\title{
FRACTIONAL LAPLACIAN PYRAMIDS
}

\author{
Ricard Delgado-Gonzalo, Pouya Dehghani Tafti, and Michael Unser \\ Biomedical Imaging Group \\ École Polytechnique Fédérale de Lausanne (EPFL) \\ CH-1015 Lausanne, Switzerland
}

\begin{abstract}
We provide an extension of the $L_{2}$-spline pyramid (Unser et al., 1993) using polyharmonic splines. We analytically prove that the corresponding error pyramid behaves exactly as a multi-scale Laplace operator. We use the multiresolution properties of polyharmonic splines to derive an efficient, non-separable filterbank implementation. Finally, we illustrate the potentials of our pyramid by performing an estimation of the parameters of multivariate fractal processes.
\end{abstract}

Index Terms - polyharmonic splines, multiresolution analysis, Laplacian pyramids, fractals.

\section{INTRODUCTION}

The Laplacian operator has a special status in image processing due to its invariances with respect to three fundamental coordinate transformations: translation, dilation and rotation. Such invariances appear naturally in real-world images, and have also been noted in mammalian visual systems [1]. It can be shown that, under appropriate assumptions, the complete class of such invariant operators reduces to the family of fractional Laplacians $(-\Delta)^{\frac{\gamma}{2}}$ with $\gamma$ in $\mathbb{R}_{+}$. There fractional Laplacians are isotropic differential operators of order $\gamma$ with the following Fourier domain definition in the sense of distributions

$$
(-\Delta)^{\frac{\gamma}{2}} f(\boldsymbol{x}) \stackrel{\mathcal{F}}{\longleftrightarrow}\|\boldsymbol{\omega}\|^{\gamma} \hat{f}(\boldsymbol{\omega}) .
$$

In this paper, we propose an extension of the $L_{2}$-spline pyramid [2], in which we replace classical polynomial splines by polyharmonic splines associated with fractional Laplacians. Our approach is applicable in an arbitrary number of dimensions and can be efficiently implemented with non-separable digital filters. We specify the corresponding error pyramid and prove that it implicitly corresponds to a wavelet analysis [3] where the basis functions are smoothed versions of the fractional Laplacian defined above. The proposed construction, therefore, behaves exactly like a Laplacian pyramid; while this behavior is only approximate in Burt and Adelson's landmark paper on multiresolution image processing [4]. We point out that our pyramid has better energy compaction due to the fact that it is built around a projector, and that it is well suited to the analysis of fractal-like processes.

\section{MULTIRESOLUTION POLYHARMONIC SPLINES}

The natural spline functions associated with the (fractional) Laplacian operator are Duchon's thin-plate splines and their polyharmonic

This work was supported in part by the Swiss National Science Foundation (under grant 200020-109415) and SystemX research consortium. extensions $[5,6]$. By definition, these functions are distributional solutions of the operator equation

$$
(-\Delta)^{\frac{\gamma}{2}} s(\boldsymbol{x})=\sum_{\boldsymbol{k} \in \mathcal{L}} c[\boldsymbol{k}] \delta\left(\boldsymbol{x}-\boldsymbol{x}_{\boldsymbol{k}}\right)
$$

where $\delta(\boldsymbol{x})$ is Dirac's delta distribution, $\boldsymbol{x}_{\boldsymbol{k}}$ 's are the spline knots, and $c[\boldsymbol{k}]$ 's are the free parameters of the model. By integration, one shows that these splines can be expressed as linear combinations of $\boldsymbol{x}_{k}$-translates of the Green's function of $(-\Delta)^{\frac{\gamma}{2}}$-also known as radial basis functions-plus a polynomial component from the nullspace of the operator. These thin-plate splines are commonly used for non-rigid registration of images; however, they are very cumbersome to implement numerically.

Fortunately, when the set of spline knots forms a uniform grid, the resulting cardinal polyharmonic splines admit a simple representation in terms of B-spline-like shift-invariant basis functions $[6,7]$. In particular, for $\gamma>d / 2$, a cardinal spline function $s(\boldsymbol{x})$ with knot spacing $T$ admits the following Shannon-like representation

$$
s(\boldsymbol{x})=\sum_{\boldsymbol{k} \in \mathbb{Z}^{d}} s(T \boldsymbol{k}) \phi_{\gamma}\left(\frac{\boldsymbol{x}-\boldsymbol{k} T}{T}\right)
$$

where $\phi_{\gamma}$ is the polyharmonic spline interpolator of order $\gamma$ over the $\mathbb{Z}^{d}$ grid; i.e., the unique polyharmonic spline that satisfies the interpolating condition $\phi_{\gamma}(\boldsymbol{k})=\delta_{\boldsymbol{k}}$ ( $\delta_{\boldsymbol{k}}$ is Kronecker's delta). The main feature of (3) is that $s(\boldsymbol{x})$ is uniquely specified by its samples on the uniform grid $T \mathbb{Z}^{d}$.

The function $\phi_{\gamma}$ has the following Fourier domain expression:

$$
\hat{\phi}_{\gamma}(\boldsymbol{\omega})=\frac{1}{1+\sum_{\boldsymbol{k} \in \mathbb{Z}^{d} \backslash\{\mathbf{0}\}}\left(\frac{\|\boldsymbol{\omega}\|}{\|\boldsymbol{\omega}+2 \pi \boldsymbol{k}\|}\right)^{\gamma}} .
$$

The above formula is an extension to fractional orders of an original result due to Madych and Nelson [6].

Cardinal polyharmonic splines satisfy dyadic scaling relations and, therefore, provide an ideal setting for constructing a multidimensional multi-resolution analysis. In particular, the interpolator $\phi_{\gamma}$ fulfills the 2 -scale relation

$$
\phi_{\gamma}(\boldsymbol{x} / 2)=\sum_{\boldsymbol{k} \in \mathbb{Z}^{d}} h_{\gamma}[\boldsymbol{k}] \phi_{\gamma}(\boldsymbol{x}-\boldsymbol{k}),
$$

where the filter $h_{\gamma}$ is specified by its frequency response

$$
H_{\gamma}\left(\mathrm{e}^{\mathrm{j} \boldsymbol{\omega}}\right)=2^{d} \frac{\hat{\phi}_{\gamma}(2 \boldsymbol{\omega})}{\hat{\phi}_{\gamma}(\boldsymbol{\omega})}=2^{d} \frac{\sum_{\boldsymbol{k} \in \mathbb{Z}^{d}}\|\boldsymbol{\omega}+2 \pi \boldsymbol{k}\|^{-\gamma}}{\sum_{\boldsymbol{k} \in \mathbb{Z}^{d}}\|\boldsymbol{\omega}+\pi \boldsymbol{k}\|^{-\gamma}},
$$

which is obviously $2 \pi$ periodic. 
Consequently, one can construct a hierarchy of dyadic multiresolution spline spaces by way of the definition

$$
\mathcal{V}_{(n)} \stackrel{\text { def }}{=}\left\{\sum_{\boldsymbol{k} \in \mathbb{Z}^{d}} a[\boldsymbol{k}] \phi_{\gamma}\left(\cdot / 2^{n}-\boldsymbol{k}\right) \mid a \in \ell_{2}\right\}
$$

with the property that $\mathcal{V}_{(n)} \subset \mathcal{V}_{\left(n^{\prime}\right)}$ for all $n>n^{\prime}$.

The dual of the polyharmonic spline interpolator $\phi_{\gamma}$ is the unique function $\tilde{\phi}_{\gamma} \in \mathcal{V}_{(0)}$ that is biorthonormal to $\phi_{\gamma}$. In the Fourier domain, the biorthonormality condition yields

$$
\hat{\tilde{\phi}}_{\gamma}(\boldsymbol{\omega})=\frac{\hat{\phi}_{\gamma}(\boldsymbol{\omega})}{\sum_{\boldsymbol{k} \in \mathbb{Z}^{d}}\left|\hat{\phi}_{\gamma}(\boldsymbol{\omega}+2 \pi \boldsymbol{k})\right|^{2}}=\frac{\hat{\phi}_{2 \gamma}(\boldsymbol{\omega})}{\hat{\phi}_{\gamma}(\boldsymbol{\omega})},
$$

where we have used the specific form of the interpolator given by Eq. (4) to establish the second equality.

Translates of $\tilde{\phi}_{\gamma}$ span the same space as those of $\phi_{\gamma}$. The biorthonormality condition of the dual basis can be used to formulate orthogonal projection onto $\mathcal{V}_{(n)}$. Specifically, let $f$ be an $L_{2}\left(\mathbb{R}^{d}\right)$ function; then the projection of $f$ onto $\mathcal{V}_{(n)}$ can be written as

$$
\mathcal{P}_{n} f(\boldsymbol{x}) \stackrel{\text { def }}{=} \sum_{\boldsymbol{k} \in \mathbb{Z}^{d}} 2^{-n d}\left\langle f, \tilde{\phi}_{\gamma}\left(\cdot / 2^{n}-\boldsymbol{k}\right)\right\rangle_{L_{2}} \phi_{\gamma}\left(\boldsymbol{x} / 2^{n}-\boldsymbol{k}\right) .
$$

Since the $\mathcal{V}_{(n)}$ spaces are nested, successive projections at different scales can be reordered and simplified in standard wavelet-theory fashion. That is, $\mathcal{P}_{n^{\prime}} \mathcal{P}_{n} f=\mathcal{P}_{n} \mathcal{P}_{n^{\prime}} f=\mathcal{P}_{n} f$ for $n^{\prime} \leq n$.

\section{THE POLYHARMONIC PYRAMID}

In this section we shall expose the existing relationship between the polyharmonic error pyramid and the fractionally iterated Laplacian operator.

The polyharmonic error pyramid is defined as a succession of functions each corresponding to the difference between two orthogonal projections of consecutive resolution. Therefore, the $n$-th level of the pyramid for $n \in \mathbb{Z}$ is expressed as

$$
\mathcal{P}_{n-1} f-\mathcal{P}_{n} f=\left(\mathcal{I}-\mathcal{P}_{n}\right) \mathcal{P}_{n-1} f,
$$

where $\mathcal{I}$ is the identity operator and where the right-hand side follows from the fact that $\mathcal{P}_{n} \mathcal{P}_{n-1}=\mathcal{P}_{n}$.

[The "Giza"1 ] Theorem. The polyharmonic error pyramid behaves like a multi-scale (fractional) Laplacian operator. Specifically, for $\gamma>d$,

$$
\begin{aligned}
\mathrm{fLP}_{n}[\boldsymbol{m}] & \left.\stackrel{\text { def }}{=}\left(\mathcal{I}-\mathcal{P}_{n}\right) \mathcal{P}_{n-1} f(\boldsymbol{x})\right|_{\boldsymbol{x}=2^{n} \boldsymbol{m}} \\
& =\left.(-\Delta)^{\frac{\gamma}{2}}\left[2^{n(\gamma-d)} \eta_{\gamma}\left(\cdot / 2^{n}\right) * f\right](\boldsymbol{x})\right|_{\boldsymbol{x}=2^{n} \boldsymbol{m}},
\end{aligned}
$$

where $\eta_{\gamma} \in L_{2}\left(\mathbb{R}^{d}\right)$ is a rescaled smoothing kernel that is characterized by its Fourier transform

$$
\hat{\eta}_{\gamma}(\boldsymbol{\omega})=\frac{1}{\|\boldsymbol{\omega}\| \gamma} \sum_{i=0}^{1}(-1)^{i+1} \frac{\hat{\phi}_{2 \gamma}\left(\boldsymbol{\omega} / 2^{i}\right)}{\hat{\phi}_{\gamma}\left(\boldsymbol{\omega} / 2^{i}\right)}<\frac{C_{\gamma}}{(1+\|\boldsymbol{\omega}\|)^{2 \gamma}},
$$

with $\hat{\eta}(\mathbf{0}) \neq 0$.

\footnotetext{
${ }^{1}$ In honor of the Great Pyramid of Giza, Built c. 2560 B.C., which is the oldest and largest of the three pyramids in the Giza Necropolis.
}

This theorem states how to compute a sampled version of the pyramid by applying the fractional Laplacian to a smoothed version of the image. Since $\hat{\eta}_{\gamma}(\mathbf{0}) \neq 0$ and $\lim _{\|\boldsymbol{\omega}\| \rightarrow \infty} \hat{\eta}_{\gamma}(\boldsymbol{\omega})=0$, the function $\eta_{\gamma}\left(\cdot / 2^{n}\right)$ acts like a low-pass filter with a bandwidth that is inversely proportional to the scale. Thus, the values of the pyramid at a certain level $n$ can be understood as the $\gamma / 2$-iterated fractional Laplacian of a bandlimited approximation of the signal. Therefore, from now on, we shall refer to the pyramid defined in (8) as the fractional Laplacian pyramid (fLP).

Note that this Laplacian-like behavior also exists over a finer grid, but its formulation requires some modification of $\eta_{\gamma}$. In particular, we can show that for uniform grids where the sampling step is $2^{n^{\prime}}$ times smaller than the sampling step of the theorem, the samples can be separated over different cosets with one single kernel acting on each coset.

\section{IMPLEMENTATION}

Because of the multi-scale properties of polyharmonic spline interpolators, it is possible to implement the corresponding projectors using a combination of non-separable digital filtering and sampling rate conversions. The proposed implementation falls within the framework of perfect reconstruction filterbanks and is summarized in Fig. 1. The synthesis filter is given by (6) while the dual analysis filter can be shown to be

$$
\tilde{H}_{\gamma}\left(\mathrm{e}^{\mathrm{j} \boldsymbol{\omega}}\right)=\frac{H_{2 \gamma}\left(\mathrm{e}^{\mathrm{j} \boldsymbol{\omega}}\right)}{H_{\gamma}\left(\mathrm{e}^{\mathrm{j} \boldsymbol{\omega}}\right)}
$$

using Eqs. (6) and (7).

Computations are all made in the Fourier domain using mirror boundary conditions. The delicate step is the precalculation of $H_{\gamma}\left(\mathrm{e}^{\mathrm{j} \boldsymbol{\omega}}\right)$ and $H_{2 \gamma}\left(\mathrm{e}^{\mathrm{j} \boldsymbol{\omega}}\right)$, which can be done efficiently thanks to a recently published result that exploits the connection between these frequency responses and the Epstein zeta function [8]

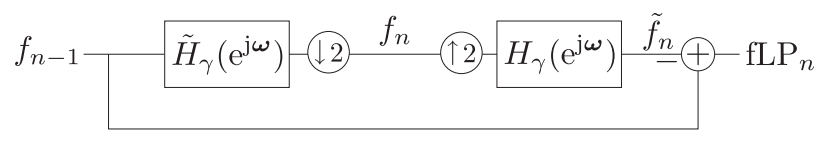

Fig. 1. Implementation of the $n$-th level of the fLP with nonseparable digital filters.

In Fig. 1, the projection of a discrete image is denoted by $f_{n}[\boldsymbol{k}]=\mathcal{P}_{n} f\left(2^{n} \boldsymbol{k}\right)$ for $\boldsymbol{k} \in \mathbb{Z}^{d} . f_{0}$ is the discrete image that corresponds to the function $f$ evaluated at the multi-integers. We also define $\tilde{f}_{n}$ as the discrete image that corresponds to the expansion of $f_{n}$ into the corresponding space at scale $n-1$. Note that because of the interpolation property of our splines, we can express this discrete image as follows: $\tilde{f}_{n}[\boldsymbol{k}]=\mathcal{P}_{n} f\left(2^{n-1} \boldsymbol{k}\right)$, for $\boldsymbol{k} \in \mathbb{Z}^{d}$.

\section{APPLICATION}

\subsection{Multiresolution analysis}

We first present some experimental results comparing the Burt and Adelson Laplacian pyramid (LP) and the fractional Laplacian pyramid (fLP) described in (8) in terms of energy compaction over 2D images.

Pyramid decompositions of Lenna $(512 \times 512)$ are shown in Fig. 2 with a parameter $a=3 / 8$ for the Burt and Adelson pyra- 
mid and $\gamma=4$ for the fLP; the contrast was linearly stretched in each subband to facilitate visual comparison. In the LP, the amount of information displayed at each resolution is significant and Lenna is still recognizable. On the other hand, in the fLP we can barely distinguish Lenna from the background; only very high-frequency details are still visible in this representation.

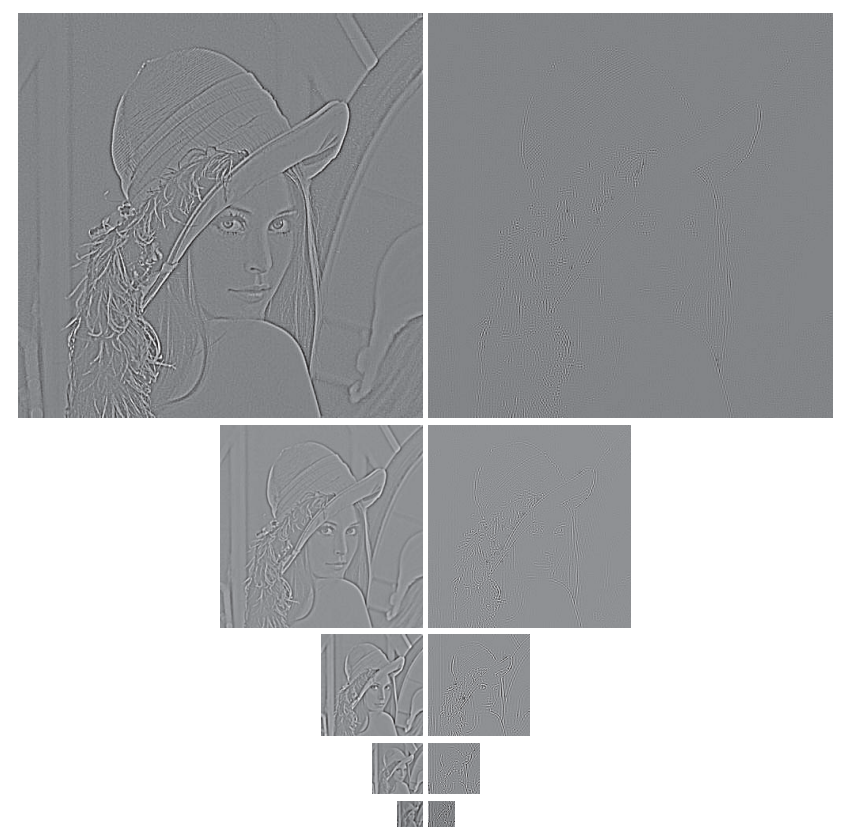

Fig. 2. Comparison of Laplacian pyramids: levels 1 to 5 of the LP (left) and the fLP (right).

In order to quantitatively compare both pyramids in terms of energy compaction we computed the root mean square (RMS) value as a measure of energy contained in each level (Fig. 3). The better performance of the fLP is particularly striking at the finest resolution where the RMS value is approximately reduced by a factor of 2 . Note that this effect is reversed at coarser scales because of the fact that the fLP has a tendency to pack the energy into the coarser levels of the pyramid.

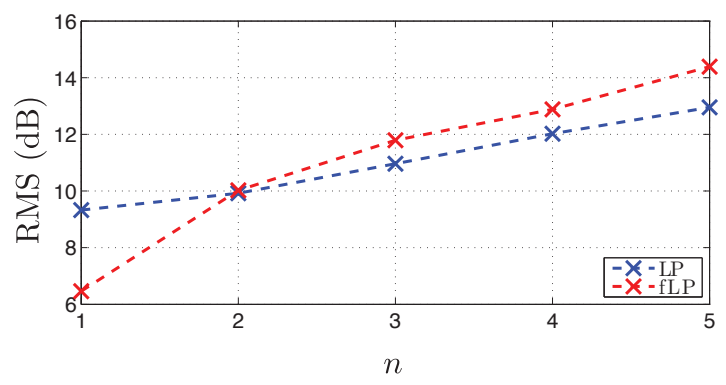

Fig. 3. Comparison of performance (in RMS terms) at successive pyramid levels for Lenna image.

\subsection{Analysis of fractal processes}

Let $\mathfrak{B}_{H}$ be a multi-variate fractional Brownian motion (fBm) field [9] with Hurst exponent $H$. Such processes are whitened through the application of a fractional Laplacian with the appropiate order $[10,11]$ :

$$
(-\Delta)^{\frac{H}{2}+\frac{d}{4}} \mathfrak{B}_{H}=\varepsilon_{H} \mathfrak{W},
$$

where $\mathfrak{W}$ is white Gaussian noise and $\varepsilon_{H}$ is a constant.

Estimation of the Hurst parameter is important in image processing due to the fact that it provides a criterion to classify different types of texture based on their second statistical moments [12]. The "Giza" theorem allows us to derive estimators for the Hurst exponent of $\mathfrak{B}_{H}$ through a multiresolution analysis. Indeed, by using (9) we show that appropriate samples of the $n$-th level of the fLP applied to a non-stationary $\mathrm{fBm}$ field $\mathfrak{B}_{H}$ are stationary Gaussian processes obtained by filtering white noise:

$$
\begin{aligned}
& \mathrm{fLP}_{n}[\boldsymbol{m}]=\left\langle\mathfrak{B}_{H},(-\Delta)^{\frac{\gamma}{2}} 2^{n(\gamma-d)} \eta_{\gamma}\left(\frac{\dot{2^{n}}}{2^{n}}-\boldsymbol{m}\right)\right\rangle \\
& =\left\langle(-\Delta)^{\frac{\gamma_{0}}{2}} \mathfrak{B}_{H},(-\Delta)^{\frac{\gamma-\gamma_{0}}{2}} 2^{n(\gamma-d)} \eta_{\gamma}\left(\frac{\cdot}{2^{n}}-\boldsymbol{m}\right)\right\rangle \\
& =\left\langle\varepsilon_{H} \mathfrak{W},(-\Delta)^{\frac{\gamma-\gamma_{0}}{2}} 2^{n(\gamma-d)} \eta_{\gamma}\left(\frac{\dot{2^{n}}}{2^{n}}-\boldsymbol{m}\right)\right\rangle \text {, }
\end{aligned}
$$

where $\gamma_{0}=H+d / 2$. In the above, the first equality is a consequence of the "Giza" theorem, the second one holds by duality, and the last follows from the definition of $\mathrm{fBm}$.

We can also establish the following exponential behavior of the variance across scale:

$$
\mathbf{E}\left\{\left|\operatorname{fLP}_{n}[\boldsymbol{m}]\right|^{2}\right\}=4^{H n} \mathbf{E}\left\{\left|\mathrm{fLP}_{0}[\boldsymbol{m}]\right|^{2}\right\} .
$$

Table 1. Performance of the fLP-based estimator for the Hurst exponent.

\begin{tabular}{|c|c|c|}
\hline True Value & mean & stdev \\
\hline \hline 0.3 & 0.294 & 0.051 \\
\hline 0.6 & 0.589 & 0.053 \\
\hline 0.9 & 0.879 & 0.054 \\
\hline
\end{tabular}

We used this result to estimate the Hurst exponent of fBm field by computing the slope of the linear regression of the log of multiscale variances. The estimation was performed on 100 instances of $512 \times 512 \mathrm{fBm}$ images for three different values of $H(0.3,0.6$ and 0.9) (see Figs. $4 \mathrm{a}-\mathrm{c}$ ) using polyharmonic splines with $\gamma=4$. Decomposition levels from 2 to 7 were used for the estimation. The average and standard deviation of the estimated values are shown in Table 1. The same multiresolution analysis was applied to a mammogram (Fig. 4d) in order to illustrate the behavior of our estimator in the case of natural fractal-like images.

\section{CONCLUSION}

Thin plate splines [5] are elegant mathematical construction but they are notoriously hard to implement because of the bad conditioning of Green's functions of the underlying Laplace operators. The proposed scheme bypasses this limitation by considering a set of Shannon-like basis functions and taking advantage of a filterbank algorithm together with an efficient Fourier-domain implementation.

We have formally shown the close relationship between the fLP and the well-known Burt and Adelson pyramid, and com- 


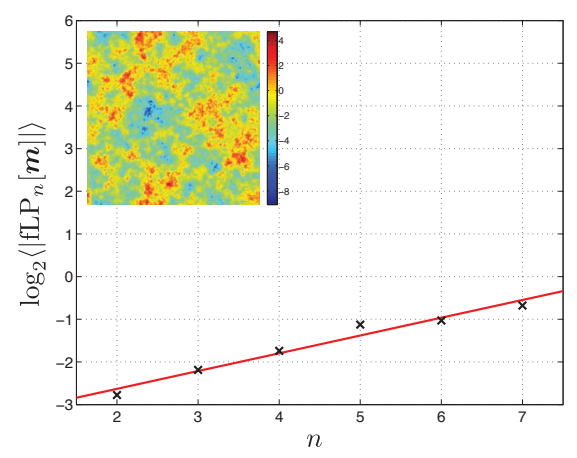

(a) fBm, $H=0.3$

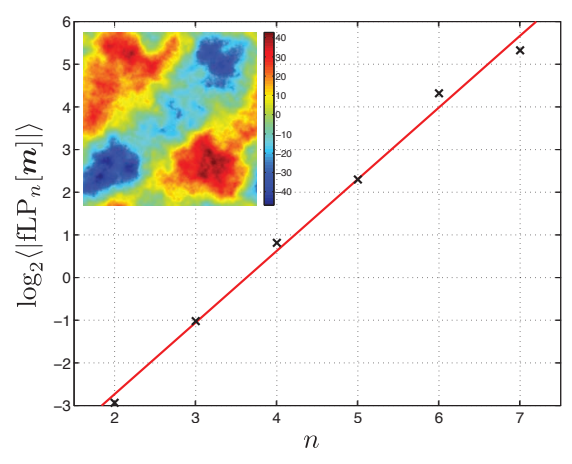

(c) $\mathrm{fBm}, H=0.9$

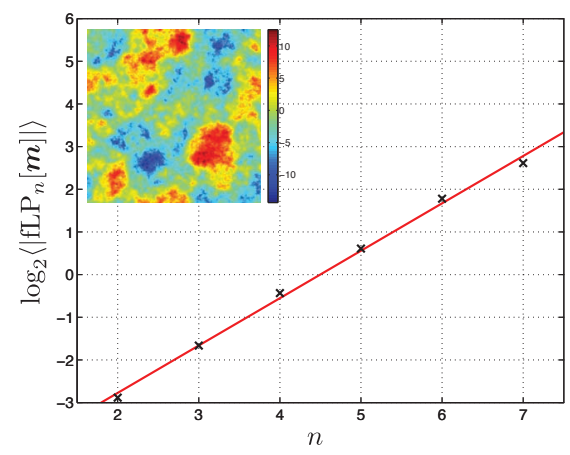

(b) $\mathrm{fBm}, H=0.6$

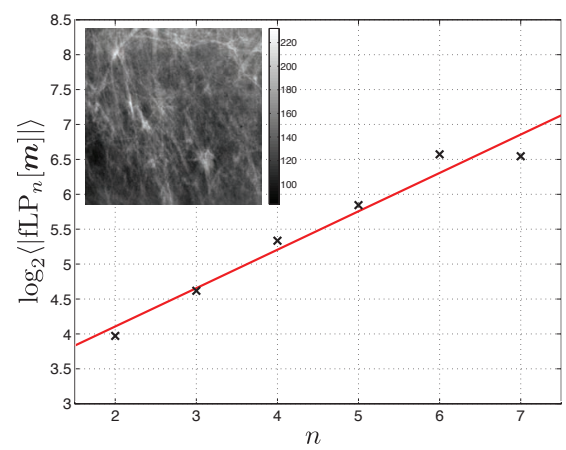

(d) Mammogram

Fig. 4. Regression plots for the estimation of the of the Hurst exponent; (a), (b), (c): single realizations of the fBm process; (d): a region of a mammogram.

pletely characterized the Laplacian behavior of the former. Since the whitening operator of a fractional Brownian motion is also a fractional Laplacian, one can exploit the Laplacian-like behavior of the fLP in order to construct multiresolution estimators of the Hurst exponent for $\mathrm{fBm}$ fields and other fractal-like images. Specifically, we used the exponential dependence of the variance of the fLP coefficients on the scale, in order to provide a log-regression based estimator of the Hurst parameter.

\section{REFERENCES}

[1] D. J. Field, "Scale-invariance and self-similar 'wavelet' transforms: an analysis of natural scenes and mammalian visual systems," in Wavelets, Fractals, and Fourier Transforms, M. Farge, J. C. R. Hunt, and J. C. Vassilicos, Eds. Oxford: Clarendon Press, 1993.

[2] M. Unser, A. Aldroubi, and M. Eden, "The $L_{2}$-polynomial spline pyramid," IEEE Transactions on Pattern Analysis and Machine Intelligence, vol. 15, no. 4, pp. 364-379, April 1993.

[3] S. Mallat, A Wavelet Tour of Signal Processing, 2nd ed. San Diego, CA: Academic Press, 1999.

[4] P. J. Burt and E. H. Adelson, "The Laplacian pyramid as a compact image code," IEEE Transactions on Communications, vol. 31, no. 4, pp. 532-540, 1983.

[5] J. Duchon, "Splines minimizing rotation-invariant semi-norms in Sobolev spaces," in Constructive Theory of Functions of
Several Variables, ser. Lecture Notes in Mathematics, A. Dold and B. Eckmann, Eds. Springer-Verlag, 1977, pp. 85-100.

[6] W. R. Madych and S. A. Nelson, "Polyharmonic cardinal splines," Journal of Approximation Theory, vol. 60, no. 2, pp. 141-156, 1990.

[7] C. Rabut, "Elementary m-harmonic cardinal B-splines," $\mathrm{Nu}$ merical Algorithms, vol. 2, pp. 39-62, 1992.

[8] Y. Barbotin, D. Van De Ville, T. Blu, and M. Unser, "Fast computation of polyharmonic B-spline autocorrelation filters," IEEE Signal Processing Letters, 2009, in press.

[9] B. B. Mandelbrot and J. W. Van Ness, "Fractional brownian motions, fractional noises and applications," SIAM Review, vol. 10, pp. 422-437, 1968.

[10] A. Benassi, S. Jaffard, and D. Roux, "Elliptic gaussian random processes," Revista matemática iberoamericana, vol. 13, no. 1, pp. 19-90, 1997.

[11] P. D. Tafti, D. Van De Ville, and M. Unser, "Invariances, Laplacian-like wavelet bases, and the whitening of fractal processes," IEEE Transactions on Image Processing, 2009, in press.

[12] T. Lundahl, W. J. Ohley, S. M. Kay, and R. Siffert, "Fractional Brownian motion: A maximum likelihood estimator and its application to image texture," IEEE Transactions on Medical Imaging, vol. 5, no. 3, pp. 152-161, Sept. 1986. 